\title{
THE \\ CLINICAL PICTURE OF STAPHYLOCOCCAL INFECTIONS IN THE NEWBORN
}

\author{
BY \\ JEAN M. CASS, M.D.* \\ (From the Babies' Hospital, Newcastle-upon-Tyne)
}

In recent years there has been increased interest in infective conditions among the newborn, and it has become evident that the conditions of institutions for the newborn are often such as to favour the occurrence and spread of infection. The staphylococcus is recognized as ubiquitous and a potentially pathogenic organism. Its constant presence in the air and on healthy hands (Devenish and Miles, 1939) and frequent presence even in the throats and milk of healthy mothers (Benians and Jones, 1929) have recently been demonstrated. This paper is an attempt to present the clinical features of the various types of disease which may occur in the newborn as a result of infection with this organism and the conditions under which such disease is liable to occur. As far as possible the material is drawn from cases personally observed at the Babies' Hospital or in the infant or maternity wards of the General Hospital, Newcastle-upon-Tyne, but the whole picture of staphylococcal infection in the newborn has necessarily been completed from published accounts of certain conditions by other authors.

\section{Blepharitis, staphylococcal ophthalmia neonatorum, and umbilical infection}

These are undoubtedly the most common forms of neonatal staphylococcal infection. They are usually mild and referred to as 'sticky eyes' or 'sticky umbilicus.' The eye infection is occasionally severe and accompanied by oedema of the eyelids and copious pus formation. When mild it usually clears within about ten days, but when severe may persist for several weeks, and in one case (R.P. see below) was followed for several months by recurrent discharge of pus from the lachrymal duct. Superficial pustules may develop on the cheek when the discharge is free, but this is commonly a surface infection only and not associated with the appearance of sepsis elsewhere. The umbilical discharge usually clears rapidly and persists only in the presence of a polyp. Infection of either the eyes or the umbilicus may be followed by signs of generalized sepsis and spread of the infection by the blood stream, of which the following case is an example.

* In receipt of a Medical Research Council Fellowship. 
Case 1. R. P. The child was healthy at birth. A purulent discharge from the eyes at the age of three days was followed by a purulent umbilical discharge after the separation of the cord. The child failed to thrive. At two months of age pyuria developed, and was followed by acute spasmodic bronchitis and septic spots. . Cough and wheezing became persistent, and there was recurrent purulent discharge from the left lachrymal duct. The gain in weight was slow, but the appetite was fairly good. From the age of six months there was irregular and unexplained pyrexia. At seven months several subcutaneous abscesses developed on the scalp and the child died apparently with an exacerbation of the chest condition. As is so often the case in infants with this type of disease post-mortem examination did not reveal any apparent naked-eye lesion in the lungs sufficient to account for death, or any localized pus.

This child evidently had an abnormally poor resistance to staphylococcal infection. Whilst the prognosis in any case of neonatal staphylococcal infection of the eyes or umbilicus is usually good, it should be remembered that a few infants have a poor resistance and the infection may be followed by a generalized spread. This will usually be made evident in a few weeks by either failure to gain weight or further signs of sepsis.

\section{Perionychiae and septic spots}

These are common forms of neonatal sepsis and were considered by Brewer (1937) to be ' part of the ordinary work of baby routine.' They are, however, definite evidence of infection among the babies and it was noticeable in the maternity ward that after several weeks of complete freedom from any infection among the mothers or babies there would be a period of a week or so in which several infants would have a few septic spots or perionychiae. These periods of infection often coincided with the development of head colds or mild sore throats among the mothers, but it was often not the child of the infected mother who developed the sign of infection, and it would appear that there existed at these times a high carrier rate among the occupants of the ward. Small perionychiae appeared to be of no significance. One infant had a severe septic thumb with swelling of the whole terminal phalanx and pus round the base of the nail, but there was no disturbance of general health or of weight gain, and no further sign of sepsis developed during the next ten weeks. It was thought that this was probably due to a high degree of immunity in the child and the rapid localization of the infection, and that the lesion was potentially dangerous. In 1925 Castle reported the case of an infant who developed a festered thumb at the age of nine days. This was followed by septic spots, otitis media, an abscess in the temporal region, and a series of subcutaneous abscesses. There was pyrexia but no disturbance of general health and the child ultimately recovered. Blood culture gave a pure growth of staphylococci on two occasions.

An attempt was made to determine on clinical grounds whether each type of septic spot was of grave or little significance. When the small and completely superficial pustule was the only evidence of sepsis, it was not followed by any other sign of sepsis. In two cases isolated flaccid bullae which contained frank pus showed no tendency to spread and on desquamation left dry skin which rapidly healed. They appeared to be purely superficial and of much less grave import than the dome-shaped bulla with clear straw coloured contents 
which appears in pemphigus. When multiple and confluent, however, these flat bullae with purulent contents may be followed by deeper septic lesions, although the bullae themselves heal rapidly. As opposed to these lesions the septic spot which is really a subcutaneous abscess, and the diffuse dull red papular or petechial rash of generalized sepsis are of grave significance, because they denote a blood stream infection. Some infants escape further evidence of infection as did the child with the severe septic thumb, but a subcutaneous abscess in the newborn is a potentially dangerous lesion.

Case 2. K. H. The child was healthy at birth. At the age of eight days it developed a septic spot on the scalp. For a month fresh spots appeared almost daily, and although most were superficial a few were described as being like small abscesses. At five weeks both ears began to discharge. When seen at the age of two months the left ear was discharging profusely and the pus yielded numerous colonies of staphylococcus aureus and several colonies of haemolytic streptococci. A post-auricular abscess and a small abscess on the cheek were both present and due to staphylococcus aureus infection. The general condition was fairly good and improved steadily.

The following case illustrates the significance of a generalized papular or petechial rash in the newborn.

Case 3. J. P. The child was healthy at birth. There was mild blepharitis with a little purulent discharge for a few days after birth. At ten days the child developed a generalized dull red papular and petechial rash. The mouth and tongue were inflamed and dry, and the motions were green and choppy. The temperature was between $98.2^{\circ} \mathrm{F}$. and $101^{\circ} \mathrm{F}$., but there was no obvious septic lesion until a week later when a small subcutaneous abscess (staphylococcus aureus) developed in the thigh. The temperature fell to normal, the rash faded and the abscess was aspirated but the child lost weight steadily and was very ill. At the age of five weeks she was small and wizened. The skin was dry and loose and the deep subcutaneous fat had been lost. She was taking her feeds fairly well, and there was no vomiting, diarrhoea or sign of local sepsis. During the next week a small abscess developed in the left axilla and at the same time the general condition improved. A diffuse papular rash appeared several times during the next few months, but her general condition improved steadily, there was no further pus formation and at five and a half months she weighed sixteen pounds.

\section{Pemphigus neonatorum, exfoliative dermatitis neonatorum and Ritter's disease}

Many cases and outbreaks of skin lesions in the newborn have been described in the past few years under the above headings. Ritter described the condition which bears his name as non-contagious, but as it was endemic in the institution in which he observed it and he personally observed 297 cases (Guy and Cohen, 1929) this was probably not the case, and it is now generally agreed that the three conditions are probably due to the same infection. Staphylococcus aureus has been the causative organism in all the outbreaks recently described, but an isolated case reported by Guy and Cohen was attributed to b.pyocyaneus infection. The essentials of the recently reported outbreaks are as follows.

Most institutional outbreaks occurred either in the presence of a skin lesion in one of the mothers or staff (Falls, 1917 ; McCandlish, 1925 ; Benians and 
Jones, 1929) or when the institution was overcrowded and understaffed (Brewer, 1937). In some cases the outbreaks were mild and easily controlled (Brewer, Benians and Jones), in one case it was prolonged for eight months in spite of vigorous efforts to stop it, and Falls reported an outbreak in which healthy mothers carried the infection from the infected institution to infants over two years of age in another institution. In all outbreaks the lesions both in adults and in the infants were vesicular rather than follicular, and in the infants were followed by desquamation. In severe cases desquamation was preceded only by redness of the skin without vesicle formation and large areas of corium were exposed, the whole lesion resembling a burn. The desquamation was followed by rapid healing. Constitutional symptoms were usually absent even in the presence of extensive desquamation, but a few infants died apparently of exhaustion. Seeds (1929) reported a case in which miliary staphylococcal abscesses of the lung followed pemphigus neonatorum.

The following is an almost identical case.

Case 4. P. D. The infant was born healthy. At three weeks a red patch appeared on one cheek. Two days later a similar patch appeared on the other cheek. On the following day red patches appeared on the legs ; the child was fretful and developed a slight cough. There was a purulent discharge from the eyes. On the fifth day large bullae developed on the hands and feet and several smaller vesicles in the groins and on the thighs and buttocks. Desquamation occurred around the anus and the external genitalia. The cry was hoarse, there was a loose cough with upper respiratory embarrassment, and a few coarse râles were heard over the bases of the lungs. On the following day desquamation was beginning over the red indurated areas, there were several fresh bullae, and the original ones had increased in size. The gums and buccal mucosa were intensely inflamed and ulcerated and feeding was extremely difficult. Fluid from an unruptured bulla yielded staphylococcus aureus in pure culture. Death occurred on the following day, seven days after the first sign of redness on the cheeks. At post-mortem examination (Dr. G. Davison) there was no abnormality of the internal organs except for the lungs which were studded with small whitish areas rather larger than pinheads and indistinguishable by the naked eye from miliary tubercles. Histological report on a portion of the lung: "Miliary focal necrosis and abscess. Abundant masses of organisms, which are obviously etiologically related to the condition and not post-mortem growth. Organisms are cocci and more like staphylococci than streptococci. The condition, at least in the terminal stage, would seem to have been pyaemic.'

The next case, in which death was due to exhaustion and toxaemia, shows how easily pyaemia may occur when the infant is infected shortly after birth.

Case 5. A. E. Child was healthy at birth. On the third day a small blister appeared in the right groin. This burst overnight and left a raw area on the thigh. The following day a small blister appeared on the cheek, and on the fifth day after birth a similar one developed on the hand. Meanwhile the child was taking its feeds well and seemed to gain weight. There was a little regurgitation of food but no vomiting or diarrhoea. On the seventh day extensive desquamation occurred and the skin lay in loose shreds. The lips were excoriated and the mouth sore. The eyelids were slightly swollen and there was some mucopurulent discharge. There was a little moistness at the attachment of the umbilical cord. The child died on the following day. A swab taken from beneath loose skin immediately after death yielded b.coli and staphylococcus albus, and a swab taken from around the umbilical cord at the same time yielded a heavy growth of staphylococcus albus. At post- 
mortem examination no significant abnormality of any organ was observed. Portions of lung, liver, spleen, kidneys and suprarenal were examined histologically with negative results. The following was the report on the umbilical cord, umbilicus, and vessels : 'A large abscess impinging on and actually entering a blood vessel which looks more like the umbilical artery than an umbilical vein. Masses of organisms are present in the pus and appear by oil immersion lens examination to be staphylococci and not streptococci.'

The following cases are interesting because they are consecutive children of the same parents, both cases of intra-uterine infection, and they illustrate the two extremes of the course of the disease.

Case 6. S. T. Normal pregnancy, delivery and puerperium. Birth weight seven pounds. Child was plump, vigorous and well developed at birth, but there was an area of desquamation on the left thigh and haemorrhagic blisters were present on the fingers. When admitted to hospital twelve hours after birth the whole of the skin was bright red, there were areas of desquamation on the thigh, fingers, chest and several dome-shaped bullae with clear serous or blood-stained fluid were present on the hands. On the following day the bullae contained haemorrhagic, purulent fluid which contained many diplococci. On culture staphylococcus albus and aureus were obtained. During the first month fresh superficial bullae with clear contents developed daily and were followed by desquamation. Large areas of desquamation appeared also without previous bulla formation and exposed freely bleeding corium which in some places discharged pus on the surface. Healing occurred rapidly from the edges of the desquamated areas. The child's weight remained stationary at about six pounds twelve ounces, and the child was vigorous and took its feeds well. The temperature ranged between $98^{\circ} \mathrm{F}$. and $100^{\circ} \mathrm{F}$. There was no cough or pyuria. At five weeks of age the temperature was between $100^{\circ} \mathrm{F}$. and $102^{\circ} \mathrm{F}$. for two days. During the next week the child lost six ounces and the motions became relaxed but not frequent, and there was a slight cough. From this time the bulla formation and desquamation became less extensive. At eight weeks of age pitting oedema developed in the legs and back, followed by oedema of the face and ascites. The weight rose to seven pounds three ounces. At this time a small subcutaneous abscess developed on the arm. The oedema passed off and the weight fell to six pounds twelve ounces. The child remained vigorous and had a good appetite. She was at this time ten weeks old and had presumably grown in spite of the persistent sepsis, because, although still the same weight as at birth, she was losing subcutaneous fat, her face was pinched and her body and limbs were small. At twelve weeks an abscess developed on her head. From this time she began to lose ground, although the weight remained stationary until about a week before death. The appetite became poor, all the subcutaneous fat was lost and the child became less vigorous. The temperature ranged between $98^{\circ} \mathrm{F}$. and $100 \cdot 6^{\circ} \mathrm{F}$. A few fresh bullae and subcutaneous abscesses developed. During the last few days before death the temperature rose to $104^{\circ} \mathrm{F}$. Death occurred at the age of seventeen weeks. On post-mortem examination, apart from the skin lesions and total absence of fat, no abnormality was discovered.

Case 7. E. T. Sister of case 6, was born fifteen months later, after normal pregnancy, delivery and puerperium. The child was well nourished and vigorous at birth but the whole of the intact skin was bright red. Large areas of desquamation were present, the skin lying in loose shreds and exposing the corium. The lips were excoriated. The child was admitted to hospital four hours after birth. The temperature was normal. Further desquamation occurred and the child rapidly lost strength. Death occurred on the third day 
after birth after a rise of temperature to $103^{\circ} \mathrm{F}$. Post-mortem examination was not allowed.

The above cases were nursed under barrier conditions and no spread of the infection occurred.

\section{Staphylococcal osteomyelitis}

This is an unusual but not rare form of neonatal sepsis. Both Green (1935) and Dilliehunt (1935), when discussing osteomyelitis in infancy, quoted cases which occurred in the neonatal period. They stressed the fact that the condition is locally relatively benign and that relatively conservative treatment suffices. In two cases seen at the Babies' Hospital in the last two years the same features were noticeable and the lesions subsided with surprisingly little or no deformity after repeated aspiration of the pus. The clinical features in these cases were strikingly different from those observed in older children in that there was practically no systemic disturbance. The mothers noticed swellings over the bones and joints of their apparently healthy infants and sought advice. In both cases the pus had ruptured into the joint when the child was first seen.

Although several bones were affected, the course of the disease was practically apyrexial, there was no loss of weight or reason for anxiety in the general condition and the lesions healed rapidly. The cases were fully reported with $\mathrm{x}$-ray plates in this journal (Cass, 1940).

\section{Staphylococcal pneumonia}

Aerogenous staphylococcal pneumonia among the newborn is an interesting though rarely recognized condition.

Smith (1935) described an outbreak of four cases in a maternity hospital. There had been several cases of breast abscess among the mothers and septic spots among the babies in the three weeks immediately preceding the outbreak. During and immediately after the outbreak eleven infants were isolated on account of mild catarrh of the upper respiratory passages, and nasal and throat swabs from these and from apparently healthy infants who were not in isolation gave a high proportion of profuse growths of staphylococcus aureus, indicating a widespread staphylococcus aureus carrier condition among the infants. Among ten fatal cases of staphylococcal pneumonia reported by Macgregor (1936), one was in a premature child sixteen days old. In all the cases the clinical picture is striking. An apparently healthy infant suddenly becomes dyspnoeic and feverish. Blood stained fluid is coughed up, vomited, or discharged from the nose. Signs of pneumonia develop and the child dies between eighteen and forty-eight hours after the first sign of illness. The post-mortem findings are equally characteristic. Blood-stained serous fluid is present in one pleural sac, subpericardial haemorrhages or bloodstained fluid in the pericardial sac, and either a portion or the whole of one lobe is sharply defined, haemorrhagic and consolidated. The remaining lung tissue appears healthy. Blood may be present in the gastrointestinal tract. Histological examination of the affected lung tissue reveals consolidation with much haemorrhage and exudate and large clumps of staphylococci. Abscess formation is evidently extremely rapid and was starting in a case reported by Smith (1935) in which death occurred thirteen hours after the onset of symptoms.

There is no clear description of a case of staphylococcal pneumonia in the newborn followed by recovery. Kanof et al. (1939) reported twelve cases of primary and eleven of secondary or pyaemic staphylococcal pneumonia, of 
which nine and five respectively occurred under one year of age. No neonatal cases are specifically mentioned and a clinical picture of the disease at this age is not given, but the clinical picture of the cases as a whole was similar to that of pneumococcal pneumonia except for the early and frequent occurrence of empyema in the staphylococcal cases. The mortality under one year of age, was 75 per cent. in the primary and 100 per cent. in the secondary cases. Macgregor (1936) describes similar cases in young infants, but all were fatal.

The following case was recently seen in this hospital.

Case 8. L. B., aged ten weeks, weighing $8 \mathrm{lb} .14 \mathrm{oz}$., was stated to have been pale and thin from birth. Pyuria and infantile eczema were present. Ten days after admission the temperature rose to between $101^{\circ} \mathrm{F}$. and $103^{\circ} \mathrm{F}$. and the child developed a cough and signs of bronchitis. Five days later 40 c.c. of pus containing staphylococcus aureus were aspirated from the left pleural cavity. In the following six weeks 361 c.c. were aspirated, the temperature gradually fell, and the child made a complete recovery.

Had the lung lesion not been followed by an empyema in this case, it would not have been diagnosed as staphylococcal pneumonia. It is possible that staphylococcal pneumonia can be a relatively mild condition in the newborn and is then unrecognized.

\section{Staphylococcal meningitis}

This appears to be a rare condition in the newborn. Craig (1936) reported twenty-one cases of neonatal meningitis. Of these, three were due to a pure staphylococcus aureus infection and three to staphylococcus aureus in combination with b.coli or streptococci. All the cases were fatal. Fifteen of the twenty-one infants were premature and although there is no note of the length of gestation in the staphylococcal cases it is clear that feeble and premature infants are particularly liable to develop meningitis in the presence of sepsis. The diagnosis of meningitis in the newborn is evidently difficult and in only four cases was it made before death. The most constant clinical features were poor appetite, increasing weakness and stationary or falling weight. Hippocratic facies, signs of cerebral irritation such as mental restlessness, darting movements of the tongue, nystagmus, unequal pupils and exaggerated reflexes developed later in some cases and suggested the need for a lumbar puncture, without which the diagnosis could not be made. Localized or general convulsions occurred in ten cases ; neck rigidity and Brudzinski's sign in only two cases. Sponginess of the fontanelle was present in nine cases. Vomiting was unusual and not projectile. Fever was usual but not constant. The pulse was in most cases rapid and almost imperceptible. In the three pure staphylococcal infections death occurred fifteen, ten and seven days after birth. In one a dorsal meningo-myelocele was present and the condition was diagnosed during life. In one there was purulent conjunctivitis with corneal ulceration, and staphylococcus aureus was isolated from the eye discharge during life. In the third case an extensive pustular skin eruption and pneumonia were present before death and at autopsy staphylococcal lung abscesses were found.

Bloch and Pacella (1938) reported a case of staphylococcal meningitis in a seventeen-day-old infant successfully treated with sulphanilamide. 
The child had an occipital meningocele removed at the age of six days. The post-operative course was uneventful, but at the age of sixteen days the infant became fretful and refused its feeds. Three generalized convulsions occurred. When seen thirty-six hours later, the temperature was normal and there were no abnormal neurological signs. A swelling was present under the operation scar and when the scab was removed a thick layer of pus containing many staphylococci was exposed. The cerebrospinal fluid was under slightly increased pressure and was cloudy. Intrathecal and oral sulphanilamide were given, the temperature subsided on the ninth day, the cerebrospinal fluid became sterile on the eleventh day, the superficial infection of the meningocele subsided and recovery occurred without complications.

This case differs from those reported by Craig in that the meninges were infected by local spread rather than by the blood stream, and circumstances suggested the diagnosis in the early stages of the disease before neurological signs had developed and the cerebrospinal fluid became purulent.

It appears that in any premature and feeble infant, or one with a congenital abnormality of the meninges, in whom there is evidence of staphylococcal infection elsewhere, weakness, loss of weight and refusal of feeds (which as already stated above, is an unusual symptom in staphylococcal infection even when severe and accompanied by a fall in weight) should suggest meningitis. Lumbar puncture in the early stages may lead to an early diagnosis and successful treatment. Evidence of cerebral irritation may develop later but the classical signs of meningitis are usually absent throughout the disease.

\section{Staphylococcal septicaemia}

In recent years there have been many reports of neonatal staphylococcal septicaemia. It is evident that many of the conditions described above are actually manifestations of septicaemia and the clinical diagnosis can be made without bacteriological confirmation. It is interesting, however, to review the clinical conditions in which the bacteriological diagnosis has been made.

Castle reported a case, already mentioned, of multiple subcutaneous abscesses in which two positive blood cultures were obtained. In 1933 Dunham reported eleven cases of staphylococcal septicaemia in which the diagnosis was made on positive blood cultures. Three recovered and eight died. The commonest clinical feature was pyrexia at some stage of the disease, present in ten cases. Jaundice, together with an enlarged spleen, fever and haematuria, was the presenting symptom in three cases. In two of these no frank sepsis manifested itself throughout the illness. In three others there was an indefinite clinical picture of pallor, cyanosis, fever and dehydration without frank sepsis. In these five cases fever was the only definite evidence of infection and the diagnosis of septicaemia could not be made on clinical grounds alone. In all the other cases there was clinical evidence of blood stream infection such as a petechial rash, otitis media, multiple abscesses, osteomyelitis or meningitis. Mount (1935) reported a case of staphylococcal septicaemia with positive blood culture. Low fever, a deep abscess in the arm with pus in the elbow joint, and abdominal distension were the chief clinical features. In contrast to these cases in which a positive blood culture has confirmed the clinical diagnosis or provided a diagnosis when the clinical signs were indefinite, is the case reported by Seeds and quoted above, in which in spite of pemphigus neonatorum and a high temperature a blood culture was negative on the fourth day. Death 
occurred on the tenth day and multiple pyaemic abscesses in the lungs were found at autopsy.

In the light of these cases there seems to be no reason why the diagnosis of septicaemia should be limited to those cases in which it is proved bacteriologically. A petechial rash, multiple subcutaneous abscesses, any deep-seated lesion such as a deep abscess, osteomyelitis or meningitis is equally good evidence of spread by the blood stream. The adult picture of septicaemia with rigors, high fever and sweats is never seen in the newborn, but rapid loss of weight and wasting are the equivalent signs of septicaemia as opposed to bacteraemia.

\section{Staphylococcal gastro-enteritis}

There have been many reported attacks of staphylococcal food poisoning in adults during the past few years. In some it has been proved without reasonable doubt that the staphylococcus isolated from the food was responsible for the illness by the production of a similar illness in human volunteers after the ingestion of the organism or a filtrate prepared from its culture (Denison, 1936 ; Kelly and Dack, 1936). No cases occurring in infancy have been reported, but Blackman (1935) reported a fatal case in a twelve-year-old child.

The onset was sudden with vomiting, headache and fever about five hours after the last ingestion of food. The vomiting continued, diarrhoea began about eighteen hours after the onset, and the child became unconscious six hours later. On admission to hospital thirty-six hours after the onset she was comatose. The temperature was $103.4^{\circ} \mathrm{F}$., pulse rate 130 , and the respirations were rapid, deep and laboured. Haemolytic staphylococcus albus was isolated from the blood and the condition was evidently one of septicaemia rather than a purely gastro-intestinal infection at this time. That the original infection had been by the intestinal tract was, however, proved by post-mortem examination ; this revealed intense inflammation and ulceration of the jejunum and ileum, and the presence of greyish red fluid and moulds of mucosa of jejunum and ileum with fibrino-purulent exudate, pus cells and clumps of staphylococci in the lumen of the bowel. The colon was normal apart from haemorrhages into the mucosa. The other findings were those of staphylococcal septicaemia.

This picture differs from that of staphylococcal septicaemia of parenteral origin as it occurred in the Bundaberg fatalities in that the gastro-intestinal symptoms are more severe and persistent and gross changes in the gastrointestinal tract are found at post-mortem examination.

It is probable that only certain strains of staphylococci produce enterotoxin and that certain conditions of growth are necessary for its production (Dolman, 1934). This may explain the apparent infrequency of staphylococcal gastroenteritis. There seems to be no doubt that the injection of staphylococcus enterotoxin causes gastro-enteritis in susceptible children and adults. Whether or not the conditions under which the food of the newborn is prepared are ever suitable for the production of enterotoxin if the necessary strain of staphylococcus is present, and whether or not the newly born are susceptible to this toxin when ingested, are not known. By analogy an outbreak of such an infection would be expected to be explosive and limited to those infants who had partaken of the food containing the toxin. Also by analogy the illness 
should be severe and short, with vomiting, diarrhoea and toxaemia, and marked signs of enteritis should be found at post-mortem examination in fatal cases. In all reported cases staphylococci have been isolated from the food or from the post-mortem lesions. As Topley and Wilson (1936) pointed out, the staphylococcus is such a common organism that one cannot incriminate staphylococci isolated from the suspected food without first proving that they are capable of producing enterotoxin under the conditions under which the food was prepared.

It may be that with increasing awareness of the possibility of staphylococcal gastro-enteritis paediatricians may be able to diagnose the condition if it occurs in the newly born and in time define the clinical picture.

\section{Anaemia as a sign of neonatal infection}

In staphylococcal neonatal infection anaemia develops slowly and is rarely, if ever, the most striking feature of the case. In purely superficial infection it was not observed. In all the infants with signs of blood stream infection the haemoglobin fell to between 50 per cent. and 66 per cent. between the sixth and twelfth week of life, but at this stage of the disease the obvious manifestations of sepsis were still present and were more striking than the pallor. The anaemia was more severe in the cases which presented the clinical picture of septicaemia than in those of bacteraemia. In both it was orthochromic and developed slowly from birth in spite of adequate doses of ferrous, copper and manganese sulphate. When the infection had become localized and was beginning to clear up, the blood returned to normal. In one case in which iron was not administered at this stage, hypochromic anaemia developed during convalescence from the infection. In case 6, S. T., in whom the infection was never completely localized and death eventually occurred, the haemoglobin remained at 50 per cent. from the age of nine weeks until just before death at the age of seventeen weeks. It appears that in blood stream staphylococcal infection in the newborn moderate or severe anaemia usually develops slowly and cannot be prevented by the administration of iron, but when the infection has been overcome the blood will return to normal if the supply of iron is adequate. When there is failure completely to localize and overcome the infection, anaemia persists in spite of iron therapy, and it is probable that unrecognized and unlocalized infection may explain the condition of some infants under six months of age with persistent anaemia which resists treatment.

\section{Discussion}

Benians and Jones (1929) demonstrated that of healthy mothers in the puerperium 15 per cent. harboured staphylococcus aureus in their throats and 17 per cent. in the breast milk, and yet their infants came to no harm. While the ubiquity of the staphylococcus ensures that all infants brought up in their natural surroundings come into early contact with the organism, it is clear that this contact is not without risk. This raises problems of immunology which are not dealt with here. It appears that although the staphylococcus aureus is constantly present in the surroundings of the newborn, it is pathogenic to only a few infants. When it is pathogenic to one, however, its virulence is increased 
by passage so that other infants are exposed to a more virulent organism. By this means an outbreak of trivial lesions may ultimately lead to an outbreak of virulent infection, as in the cases of pneumonia reported by Smith. Therefore although it is not known why isolated infants succumb to an infection with this common organism, experience suggests that all such cases, however mild the infection, should be isolated to prevent the spread of an increasingly virulent organism and a serious type of lesion.

The specificity of the type of lesion in any one outbreak is interesting. Falls (1917) remarked that in the outbreaks of pemphigus neonatorum observed by him all the lesions were vesicular and the organism, although culturally staphylococcus aureus, tended to appear as a diplococcus on certain media. He suggested that pemphigus neonatorum is due to a specific strain of staphylococcus aureus. Benians and Jones (1929) also noticed the occurrence of vesicular lesions even among the adults infected in the outbreaks described by them, and remarked on the frequent contact of healthy infants with staphylococcus aureus but the rarity of pemphigus neonatorum. They observed no fixed differentiating characters in the organisms isolated from their cases of pemphigus neonatorum from the ordinary staphylococcus aureus. It is remarkable that aerogenous staphylococcal pneumonia has been reported after an outbreak of septic spots but not in a case of pemphigus neonatorum, although the lips and mouth are often affected early. On the other hand the outbreak of pemphigus described by Brewer (1937) was associated with a boil in a nurse and napkin rashes and perionychiae among the infants. It is not clear from the description of the lesions whether they were dome-shaped bullae with clear straw coloured contents or flat vesicles containing frank pus. As far as I have observed, the latter are much less serious and are more akin to superficial septic spots than to true bullous impetigo. In the absence of accurate knowledge of the type of lesion present in these cases, no definite conclusions can be drawn, but all the other evidence available suggests that true pemphigus neonatorum characterized by clear bullae and desquamation exposing the corium, is due to infection with a specific strain of staphylococcus aureus.

There seem to be three clinical types of response on the part of the newly born to the common early staphylococcal infections such as blepharitis and umbilical infection. The most usual is a rapid and complete recovery without any illness and sequelae. Fairly common is the second type of response in which the appetite and weight gain are normal throughout, and there is never any anxiety about the child's general condition, but many septic lesions such as abscesses, otitis media and osteomyelitis may develop in the next few months. This condition is not unlike infantile pulmonary tuberculosis after the age of six months since in both the general condition remains good in spite of an obvious lesion. Also, although the infants are liable to fresh metastatic lesions in any organ and may be left with permanent deformity if the bones are involved, the ultimate prognosis as regards life is excellent unless a rare complication such as pyaemic spread to the lungs, meningitis or secondary infection supervenes. The third type of response occurs in only a few infants in whom resistance to the infection is unusually deficient. In these a dangerous illness follows infection 
and is characterized by wasting, a toxic rash, often pyrexia and occasionally a poor appetite. Pus formation is delayed and slight and may or may not be followed by an improvement in the general condition. The condition may fluctuate for weeks or months, and death may finally occur without any apparent cause except toxaemia and with no obvious septic focus. More often after a few weeks suppuration occurs, the weight rises, the temperature falls and the general condition improves. When the child begins to thrive it is usually out of danger. A steady gain in weight in the presence of suppuration is of far better significance than stationary or falling weight with little or no suppuration.

\section{Summary}

The clinical types of staphylococcal infections in the newborn are reviewed and the significance and prognosis of each type are discussed. The potential dangers of skin lesions both to the infants affected and to those in contact with them is noted. It is found that the newborn react to staphylococcal infection in a characteristic manner, but as in adults, the infection may be apparently completely localized, bacteraemic or septicaemic in nature. The difficulty of making the diagnosis of staphylococcal meningitis and staphylococcal pneumonia during life, the relative values of clinical bacteriological signs of staphylococcal septicaemia and the absence of reported cases of staphylococcal gastro-enteritis in the newborn are discussed. Anaemia is found to be a late and relatively mild complication of staphylococcal neonatal sepsis, but it persists until the infection is completely localized and may therefore persist long after the neonatal period.

Thanks are due to Dr. J. C. Spence for the personal interest and encouragement he has given to this work and for his valuable suggestions; to all the members of the staff of the Babies' Hospital for their co-operation and interest ; to Dr. Norton, bacteriologist at the City Hospital for Infectious Diseases, Walker Gate, for all the bacteriological work ; and to Dr. Harlan, Medical Superintendent of the General Hospital, for access to the wards of that hospital.

\section{REFERENCES}

Benians, T. C. H., and Jones, B. H. (1929). Lancet, 1, 174.

Blackman, S. S. (1935). Johns Hopk. Hosp. Bull., 57, 289.

Bloch, H., and Pacella, B. L. (1938). J. Amer. med. Ass., 110, 508.

Brewer, D. (1937). Med. Off., 57, 75.

Cass, J. M. (1940). Arch. Dis. Childh., 15, 55.

Castle, W. F. (1925). Brit. J. Child. Dis., 22, 122.

Craig, W. S. (1936). Arch. Dis. Childh., 12, 171.

Denison, G. A. (1936). Amer. J. pub. Hlth., 26, 1168.

Devenish, E. A., and Miles, A. A. (1939). Lancet, 1, 1088.

Dilliehunt, R. B. (1935). Surg. Gynec. Obstet., 61, 96.

Dolman, C. E. (1934). J. infect. Dis., 55, 172.

Dunham, E. C. (1933). Amer. J. Dis. Child., 45, 229.

Falls, F. H. (1917). J. infect. Dis., 20, 86.

Green, W. T. (1935). J. Amer. med. Ass., 105, 1835.

Grubb, T. C. (1938). J. Lab. clin. Med., 23, 1150.

Guy, W. H., and Cohen, M. (1929). Arch. Derm. Syph., Chicago, 19, 425.

Kanof, A., Kramer, B., and Carnes, M. (1939). J. Pediat., 14, 712.

Kelly, F. C., and Dack, G. M. (1936). Amer. J. pub. Hlth., 26, 1077.

Macgregor, A. R. (1936). Arch. Dis. Childh., 11, 195.

McCandlish, H. S. (1925). Amer. J. Obstet. Gynec., 9, 228.

Mount, W. B. (1935). Ibid., 29, 126.

Seeds, A. E. (1929). Virginia med., 56, 530.

Smith, C. M. (1935). Lancet, 1, 1204.

Topley, W. W. C., and Wilson, G. S. (1936). The Principles of Bacteriology and Immunity, second edition, London, 1268. 